\title{
Orbital Lymphoma Presenting with Inferior Rectus Palsy
}

\author{
Jae Ho Jung ${ }^{\text {a }}$ \\ Eun Hye $\mathrm{Oh}^{\mathrm{b}}$ \\ Dong Hoon Shin ${ }^{c}$ \\ Seo-Young Choi ${ }^{\mathrm{d}}$ \\ Kwang-Dong Choi ${ }^{\mathrm{d}}$ \\ Jae-Hwan Choi ${ }^{\mathrm{b}}$ \\ aDepartment of Ophthalmology \\ Seoul National University Hospital, \\ Seoul, Korea \\ ${ }^{b}$ Departments of Neurology and \\ 'Pathology, Pusan National University \\ School of Medicine, Research Institute for \\ Convergence of Biomedical Science and \\ Technology, Pusan National University \\ Yangsan Hospital, Yangsan, Korea \\ ${ }^{d}$ Department of Neurology, \\ Pusan National University Hospital, \\ Pusan National University \\ School of Medicine and \\ Biomedical Research Institute, \\ Busan, Korea
}

Received November 14, 2018

Revised January 20, 2019

Accepted January 22, 2019

\section{Correspondence}

Jae-Hwan Choi, MD

Department of Neurology,

Pusan National University

School of Medicine,

Research Institute for

Convergence of Biomedical Science and

Technology, Pusan National University

Yangsan Hospital, 20 Geumo-ro,

Mulgeum-eup, Yangsan 50612, Korea

Tel +82-55-360-2122

Fax +82-55-360-2152

E-mail rachelbolan@hanmail.net
Dear Editor,

A previously healthy 54-year-old woman presented with the insidious onset of vertical diplopia that first appeared several months previously. She had no headache or ocular pain, fever, night sweat, or weight loss. The diplopia was aggravated while looking leftward and downward. Ophthalmic examinations revealed left eyelid swelling with mild chemosis and exophthalmos, and depression deficit of the left eye during left downward gaze (Fig. 1A). A forced duction test on the left eye indicated paralytic strabismus. The Hess test disclosed isolated inferior rectus (IR) palsy of the left eye (Fig. 1B). The findings of other neuro-ophthalmological examinations were normal. An MRI scan of the orbit revealed prominent thickening and enhancement of the left IR muscle, but there was also mild swelling of the left lacrimal gland, superior rectus (SR) muscle, and medial rectus muscle (Fig. 1C). The results for complete blood count, serum biochemistry profiles, thyroid function, and autoimmune antibodies were normal.

The patient was thought to have nonspecific orbital inflammation, and was treated with oral prednisolone at $1 \mathrm{mg} / \mathrm{kg}$ daily, which was tapered over 3 months. However, the vertical diplopia and the IR muscle enlargement did not improve. To make a correct diagnosis, we performed a biopsy of the left IR muscle via a retroseptal transconjunctival approach. Intraoperative findings revealed significant IR enlargement without muscle stiffness and infiltrations around the muscle. Histopathologically, atypical lymphoid infiltrates were present between scattered, degenerated muscular bundles (Fig. 1D). The cells were positive for CD3, CD20, and BCL2, consistent with extranodal marginal-zone lymphoma. In situ hybridization for Epstein-Barr virus-encoded RNA produced negative findings. An 18-Fluoro-deoxyglucose-PET scan showed hypermetabolism in the left IR and pectoralis muscle (Fig. 1E). Radiotherapy at 3,060 cGy in 17 fractions to the left orbit resulted in improvement in the eyelid swelling, diplopia, and ophthalmoplegia. Follow-up MRI scans showed decreases in the extent of thickening and enhancement of the left extraocular muscles (EOMs) and the lacrimal gland.

Our patient showed IR palsy due to EOM involvement in extranodal marginal-zone lymphoma. Acquired IR palsy is rare and mostly occurs with brainstem or orbital lesion. ${ }^{1}$ The IR muscle is innervated by the inferior division of the oculomotor nerve that originates from the oculomotor nucleus in the rostral part of the midbrain. Since the oculomotor nucleus and fascicles are topographically arranged in the midbrain, selective damage to the IR subnucleus or fascicle can produce IR palsy in the brainstem. These disorders include cerebral infarction, multiple sclerosis, and benign or metastatic tumors. ${ }^{1-3}$ In the orbit, on the other hand, damage to the oculomotor terminal branch supplying the IR muscle, its myoneural junction, or the IR muscle itself may produce IR palsy. This condition may be associated with orbital trauma, orbital adhesive disease, myasthenia gravis, and complications when performing ocular surgery. ${ }^{1}$ IR palsy due to a tumor is extremely rare, with only a few cases having been reported in the literature. ${ }^{2,3}$ These cases had a cavernous angioma or metastatic

@ This is an Open Access article distributed under the terms of the Creative Commons Attribution Non-Commercial License (https://creativecommons.org/licenses/by-nc/4.0) which permits unrestricted non-commercial use, distribution, and reproduction in any medium, provided the original work is properly cited. 

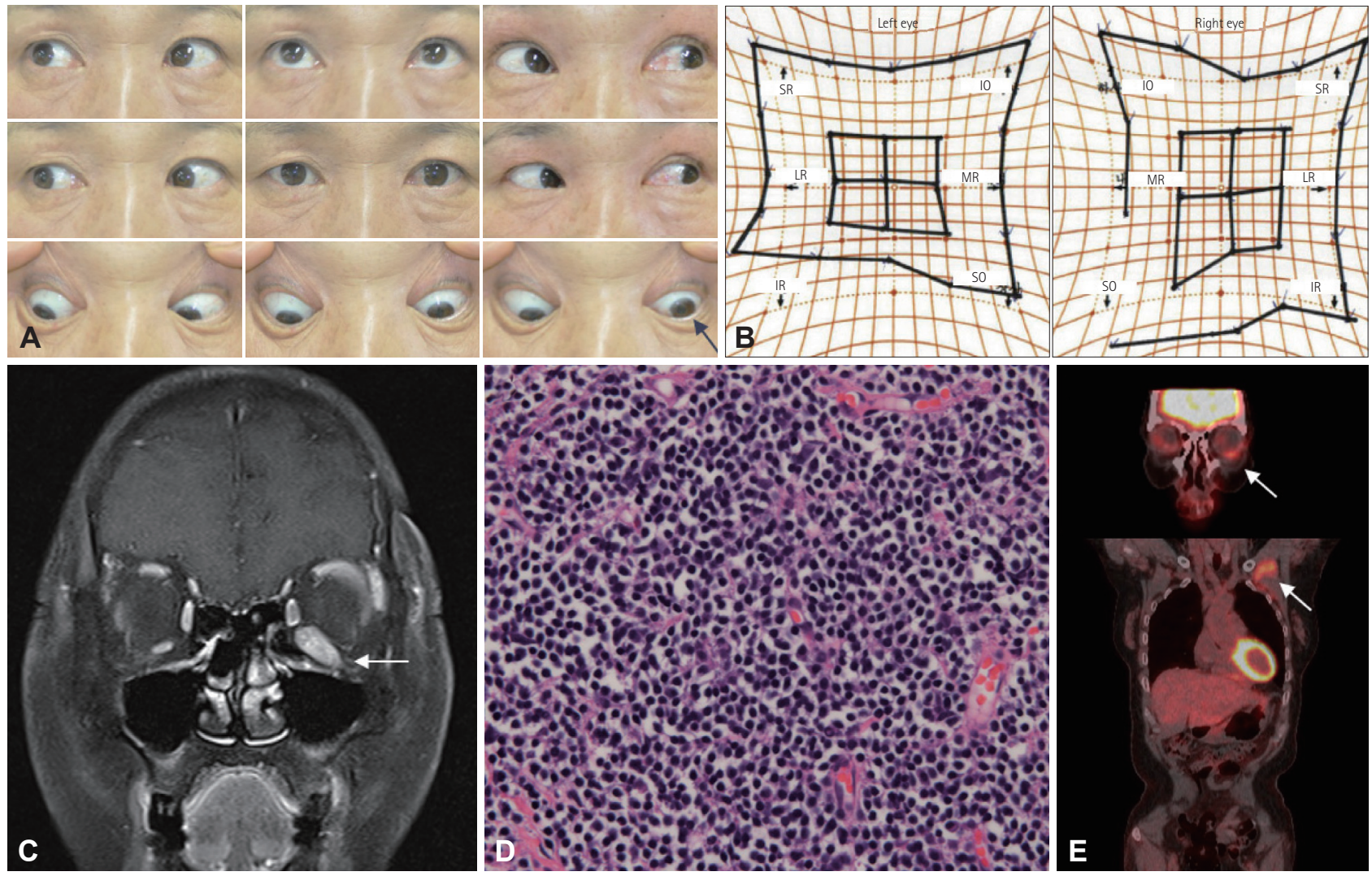

Fig. 1. Nine-gaze photograph shows the left eyelid swelling with mild chemosis, and depression deficit of the left eye in the left downward gaze (ar$\left.\operatorname{row}_{1} A\right)$, and the Hess test revealing the isolated IR palsy of the left eye (B). MRI scan of the orbit discloses a prominent enlargement of the left IR muscle (arrow, C), but also mild swelling of the left lacrimal gland, SR, and MR muscles. A section of the left IR muscle shows atypical lymphoid infiltrates with small dark nuclei and clear cytoplasm (hematoxylin \& eosin; $\times 400, \mathrm{D}$ ). An FDG-PET scan reveals hypermetabolism in the left IR and pectoralis muscle (arrows, E). FDG: 18-fluoro-deoxyglucose, 10: inferior oblique, IR: inferior rectus, LR: lateral rectus, MR: medial rectus, SO: superior oblique, SR: superior rectus.

tumor in the rostral midbrain, resulting in IR palsy by the involvement of the IR subnucleus. However, in our patient the IR muscle itself was involved by the infiltration of orbital lymphoma.

Orbital lymphoma is a rare tumor, comprising only $1 \%$ of all cases of non-Hodgkin's lymphoma. ${ }^{4}$ It is generally a lowgrade malignancy, and marginal-zone B-cell lymphoma is the most common type. The common presenting symptoms or signs are eyelid swelling, palpable eyelid mass, double vision, and proptosis. It can affect all orbital structures including the conjunctiva, eyelid, lacrimal gland, EOMs, and optic nerve. The most frequent location is the superior-lateral quadrant due to the involvement of the SR and lateral rectus muscle, whereas the IR muscle is rarely affected. ${ }^{5}$ Isolated EOM infiltration by an orbital lymphoma has rarely been reported in the literature, and this most commonly involves the SR/levator muscle complex. ${ }^{6-8}$ In our patient, the involvement of the IR muscle was more prominent than that in the other EOMs, leading to a downward gaze deficit of the affected eye. The enlargement of the IR muscle is commonly associated with thy- roid orbitopathy, but it leads to upgaze restriction due to fibrosis of the IR muscle. The forced duction test can often provide sufficient diagnostic information in this situation. Idiopathic orbital inflammation (IOI) should also be considered in the differential diagnosis of EOM enlargement. However, the muscle swelling with severe pain is more acute at the onset in IOI, and this responds rapidly to steroid with a dramatic improvement, in contrast to the observations in our patient. Although orbital lymphoma shows a good response to the radiotherapy, it can exhibit recurrence or the occurrence of systemic lymphoma. ${ }^{5}$ Thus, orbital lymphoma should be considered as a possible etiology of EOM enlargement, especially in cases that do not respond to steroids.

\section{Conflicts of Interest}

The authors have no potential conflicts of interest to disclose.

\section{REFERENCES}

1. Choi KD, Choi JH, Choi HY, Huh YE, Kim HJ, Oh SY, et al. Inferior rectus palsy as an isolated ocular motor sign: acquired etiologies and 
outcome. J Neurol 2013;260:47-54.

2. Pusateri TJ, Sedwick LA, Margo CE. Isolated inferior rectus muscle palsy from a solitary metastasis to the oculomotor nucleus. Arch Ophthalmol 1987;105:675-677.

3. Chou TM, Demer JL. Isolated inferior rectus palsy caused by a metastasis to the oculomotor nucleus. Am J Ophthalmol 1998;126:737-740.

4. Eckardt AM, Lemound J, Rana M, Gellrich NC. Orbital lymphoma: diagnostic approach and treatment outcome. World J Surg Oncol 2013; 11:73.

5. Demirci H, Shields CL, Karatza EC, Shields JA. Orbital lymphoproliferative tumors: analysis of clinical features and systemic involve- ment in 160 cases. Ophthalmology 2008;115:1626-1631.

6. Malik KJ, Berntson DG, Harrison AR. Lymphoplasmacytic lymphoma isolated to an extraocular muscle. Ophthalmic Plast Reconstr Surg 2006;22:400-401.

7. Izambart C, Robert PY, Petellat F, Petit B, Gastaud P, Lagier J, et al. Extraocular muscle involvement in marginal zone B-cell lymphomas of the orbit. Orbit 2008;27:345-349.

8. Benetatos L, Alymara V, Asproudis I, Bourantas KL. Rituximab as first line treatment for MALT lymphoma of extraocular muscles. Ann Hematol 2006;85:625-626. 